\title{
Euler-Poincaré Models of Ideal Fluids with Nonlinear Dispersion
}

\author{
Darryl D. Holm* \\ Theoretical Division and Center for Nonlinear Studies, Los Alamos National Laboratory, MS B284, \\ Los Alamos, New Mexico 87545 \\ Jerrold E. Marsden ${ }^{\dagger}$ \\ Control and Dynamical Systems, California Institute of Technology 107-81, Pasadena, California 91125 \\ Tudor S. Ratiu \\ Department of Mathematics, University of California, Santa Cruz, California 95064
}

(Received 6 January 1998)

\begin{abstract}
We propose a new class of models for the mean motion of ideal incompressible fluids in three dimensions, including stratification and rotation. In these models, the amplitude of the rapid fluctuations introduces a length scale, $\alpha$, below which wave activity is filtered by both linear and nonlinear dispersion. This filtering enhances the stability and regularity of the new fluid models without compromising either their large scale behavior, or their conservation laws. These models also describe geodesic motion on the volume-preserving diffeomorphism group for a metric containing the $H^{1}$ norm of the fluid velocity. [S0031-9007(98)06028-1]
\end{abstract}

PACS numbers: $47.10 .+\mathrm{g}, 03.40 . \mathrm{Gc}$

Linear dispersion is well known to have profound effects on wave mean flow interaction in fluids [1]. In one dimension, for example, linear dispersion may balance nonlinear steepening to produce coherent (spatially localized) structures such as solitary waves and, in special circumstances, solitons. (Solitons are solitary waves that scatter among themselves elastically - up to a phase shift.) There is a soliton-bearing equation due to Camassa and Holm $(\mathrm{CH})$ [2] that contains both linear and nonlinear dispersion, namely,

$u_{t}+2 \kappa u_{x}+3 u u_{x}-\alpha^{2} u_{x x t}=2 \alpha^{2} u_{x} u_{x x}+\alpha^{2} u u_{x x x}$,

where $u: \mathbb{R} \times \mathbb{R} \rightarrow \mathbb{R}$ is the fluid velocity in the $x$ direction and subscripts denote partial derivatives. The constants $\kappa$ and $\alpha$ have units of speed and length, respectively. The linear dispersion relation for $\mathrm{CH}$ in (1) is $\omega=2 \kappa k /\left(1+\alpha^{2} k^{2}\right)$. The $\kappa \neq 0$ solutions of $\mathrm{CH}$ are solitons which behave similarly to those for Kortwegde Vries $\left(u_{t}+2 \kappa u_{x}+3 u u_{x}+\alpha^{2} c_{0} u_{x x x}=0\right.$, with $c_{0}$ a speed). However, for $\kappa=0$, the linear dispersion in $\mathrm{CH}$ vanishes and its remaining nonlinear dynamics allows the superposition of $N$ solitons, as $u(x, t)=$ $\sum_{i=1}^{N} p_{i}(t) \exp \left(-\left|x-q_{i}(t)\right| / \alpha\right)$. These solutions possess $N$ peaks at which $u_{x}$ reflects in sign. The $N$-soliton solutions of $\mathrm{CH}$ are called peakons, and their dynamics has been well studied [2-11].

Remarkably, $\mathrm{CH}$ describes geodesic motion on the diffeomorphism group of the real line $\mathbb{R}$ for the metric given by the $H_{\alpha}^{1}$ norm of the fluid velocity, $\|u\|_{\alpha}^{2}=$ $\int_{\mathbb{R}} d x\left(u^{2}+\alpha^{2} u_{x}^{2}\right)$, with parameter $\alpha$ [12]. The proof of this property in the case $\kappa=0$ is obtained easily, by noticing that $\mathrm{CH}$ is a one-dimensional Euler-Poincaré (EP) equation [13-15],

$$
\begin{aligned}
& v_{t}+u v_{x}+2 v u_{x}=0, \\
& \quad \text { where } v \equiv \frac{\delta l}{\delta u} \quad \text { and } \quad \frac{\delta l_{\mathrm{CH}}}{\delta u}=u-\alpha^{2} u_{x x},
\end{aligned}
$$

for the Lagrangian $l$ given by the metric $l_{\mathrm{CH}}=\frac{1}{2}\|u\|_{\alpha}^{2}$. (EP equations are the Lagrangian analog of Lie-Poisson Hamiltonian equations [14].) This geodesic property readily generalizes to $n$ dimensions and thus produces an $n$-dimensional $\mathrm{CH}$ equation via the EP theory. We shall examine this later, but first we emphasize a key feature of the nonlinear dispersion in the EP equation (2) for $l=l_{\mathrm{CH}}$, namely, its Helmholtz smoothing. That is, the transport velocity $u=\left(1-\alpha^{2} \partial_{x}^{2}\right)^{-1} v$ is Helmholtz smoothed relative to the momentum $v$ for $\mathrm{CH}$. This smoothing endows $\mathrm{CH}$ with much more regularity than the Riemann equation $u_{t}+3 u u_{x}=0$, which is the EP equation for $\alpha=0$ corresponding to the $L^{2}$ norm $|u|^{2}=$ $\int_{\mathbb{R}} d x u^{2}$. The mechanism of this Helmholtz smoothing can be understood by taking the Fourier transform of Eq. (2) to find with $u_{p}=v_{p} /\left(1+\alpha^{2} p^{2}\right)$

$$
\frac{d v_{k}}{d t}+i \sum_{|n+p|=|k|} \frac{n+2 p}{1+\alpha^{2} p^{2}} v_{p} v_{n}=0 .
$$

At low wave numbers, when $|n+2 p| \approx\left(1+\alpha^{2} p^{2}\right)$, nonlinear steepening produces a forward cascade in wave number. However, at higher wave numbers, when $\mid n+$ $2 p \mid<\left(1+\alpha^{2} p^{2}\right)$, this cascade is quickly stifled by Helmholtz smoothing. Thus, the introduction of the length scale $\alpha$ and the associated Helmholtz smoothing in its nonlinear dispersion changes the shock behavior of the Riemann equation for $\alpha=0$ into the peakon behavior of the $\mathrm{CH}$ equation. And the Lagrangian of the onedimensional EP equation (2) changes from the $L^{2}$ norm 
of the fluid velocity (the kinetic energy) for $\alpha=0$ to the $H_{\alpha}^{1}$ norm.

Geophysical Applications. - The Euler-Boussinesq (EB) equations for a rotating stratified incompressible ideal fluid are [16]

$$
\begin{gathered}
\frac{d \mathbf{u}}{d t}-\mathbf{u} \times \operatorname{curl} \mathbf{R}+g b \hat{\mathbf{z}}+\frac{1}{\rho_{0}} \nabla p=0, \\
\nabla \cdot \mathbf{u}=0, \quad \frac{d b}{d t}=0,
\end{gathered}
$$

where $d / d t=\partial / \partial t+\nabla$ is the material derivative, $b$ is buoyancy, $p$ is pressure, $g$ and $\rho_{0}$ are constants, and curl $\mathbf{R}=2 \boldsymbol{\Omega}+O(\beta), \beta \ll 1$, is the Coriolis parameter, with constant angular frequency $\boldsymbol{\Omega}$. These equations are EP equations [15], namely,

$$
\frac{d}{d t} \frac{1}{D} \frac{\delta l}{\delta \mathbf{u}}+\frac{1}{D} \frac{\delta l}{\delta u^{j}} \nabla u^{j}+\frac{1}{D} \frac{\delta l}{\delta b} \nabla b-\nabla \frac{\delta l}{\delta D}=0,
$$

which are stationarity conditions for the EB Lagrangian $l_{\mathrm{EB}}(\mathbf{u}, b, D)$ given by

$$
\begin{aligned}
l_{\mathrm{EB}}= & \int d^{3} x \rho_{0} D\left(\frac{1}{2}|\mathbf{u}|^{2}+\mathbf{u} \cdot \mathbf{R}(\mathbf{x})-g b z\right) \\
& -p(D-1),
\end{aligned}
$$

for certain constrained variations and for velocity tangential to the boundary. The buoyancy $b$ and the volume element $D$ satisfy advection dynamics, $d b / d t=0$, $d D / d t=-D \nabla \cdot \mathbf{u}$ and incompressibility $(\nabla \cdot \mathbf{u}=0)$ holds when the constraint $D=1$ is applied. The Euler equations - namely, (4) without rotation and buoyancyare geodesic on the volume-preserving diffeomorphisms with the $L^{2}$ normalization, $\int d^{n} x|\mathbf{u}|^{2}$ [17]. There is a Kaluza-Klein construction in which the EB equations are also geodesic [18].

Deriving $\alpha$ models. - We consider the mean dispersive effects of internal gravity waves on the solutions of the EB equations [1]. For this, we consider an individual fluid parcel trajectory. In the presence of a wave that induces a displacement field $\alpha \boldsymbol{\xi}$, this fluid trajectory is given by $\mathbf{x}^{\xi}=\mathbf{x}+\alpha \boldsymbol{\xi}(\mathbf{x}, t)$. Here, the constant length $\alpha$ scales the amplitude of the displacement (or its amplitude-to-wavelength ratio $\alpha / L \ll 1$ in a nondimensional formulation) and the vector $\boldsymbol{\xi}$ has mean $\overline{\boldsymbol{\xi}}=0$. The corresponding fluid velocity is $\mathbf{u}^{\xi}=\mathbf{u}(\mathbf{x}+\alpha \boldsymbol{\xi})=$ $\mathbf{u}(\mathbf{x})+\alpha \boldsymbol{\xi} \cdot \nabla \mathbf{u}+O\left(\alpha^{2}\right)$. To obtain the mean dispersive effects due to internal wave displacements, we average the Lagrangian $l_{\mathrm{EB}}$ over the phase of the rapid fluctuations following the fluid parcels to find

$$
\begin{gathered}
\overline{l_{\mathrm{EB}}}=\int d^{3} x\left\{\rho_{0} D\left(\frac{1}{2} \overline{\left|\mathbf{u}^{\xi}\right|^{2}}+\overline{\mathbf{u}^{\xi} \cdot \mathbf{R}^{\xi}(\mathbf{x})}-g \overline{b^{\xi} z^{\xi}}\right)\right. \\
-p(D-1)\}+O\left(\alpha^{2} \boldsymbol{\epsilon}\right),
\end{gathered}
$$

in which we neglect corrections due to fluctuations of the volume element $D$ of order $O\left(\alpha^{2} \epsilon\right)$, where $\epsilon \ll 1$ is the ratio of time scales between fluctuations and mean quantities. (See [1,19] for discussions of such higher order corrections.) We approximate the mean kinetic energy in a Taylor expansion as follows:

$$
\begin{aligned}
\frac{1}{2} \overline{|\mathbf{u} \xi(\mathbf{x}, t)|^{2}} & =\frac{1}{2} \overline{|\mathbf{u}(\mathbf{x}+\alpha \boldsymbol{\xi}, t)|^{2}} \\
& =\frac{1}{2} \overline{|\mathbf{u}(\mathbf{x}, t)+\alpha \boldsymbol{\xi} \cdot \nabla \mathbf{u}|^{2}}+O\left(\alpha^{4}\right) \\
& =\frac{1}{2}|\mathbf{u}|^{2}+\frac{\alpha^{2}}{2} \overline{|\xi \cdot \nabla \mathbf{u}|^{2}}+O\left(\alpha^{4}\right) \\
& \approx \frac{1}{2}|\mathbf{u}|^{2}+\frac{\alpha^{2}}{2}|\nabla \mathbf{u}|^{2} .
\end{aligned}
$$

In the last step, we drop terms of order $O\left(\alpha^{4}\right)$ and assume isotropy of the rapid fluctuations, so that $\overline{\xi^{i} \xi^{j}} \approx$ $\delta^{i j}$. (This assumption may require modification near boundaries.) There is a corresponding expansion for the rotational terms. On the other hand, since the buoyancy is constant following a fluid parcel, we find $\overline{b^{\xi}(\mathbf{x})(z+\alpha \boldsymbol{\xi} \cdot \hat{\mathbf{z}})}=b(\mathbf{x}) z$. Thus, up to terms of order $O\left(\alpha^{2} \epsilon, \alpha^{4}\right)$ we have

$$
\begin{gathered}
\overline{l_{\mathrm{EB}}}=\int d^{n} x\left\{\rho _ { 0 } D \left(\frac{1}{2}|\mathbf{u}|^{2}+\frac{\alpha^{2}}{2}|\nabla \mathbf{u}|^{2}+\mathbf{u} \cdot \mathbf{R}(\mathbf{x})\right.\right. \\
\left.+\alpha^{2} \operatorname{tr}\left(\nabla \mathbf{u} \cdot \nabla \mathbf{R}^{T}\right)-g b z\right) \\
-p(D-1)\}
\end{gathered}
$$

Here, $\operatorname{tr}\left(\nabla \mathbf{u} \cdot \nabla \mathbf{R}^{T}\right)=\boldsymbol{\omega} \cdot \boldsymbol{\Omega}+O(\beta)$, where $\boldsymbol{\omega}=$ curl $\mathbf{u}$. We shall omit the $\alpha^{2} \boldsymbol{\omega} \cdot \boldsymbol{\Omega}$ term in what follows, as well as the order $O\left(\alpha^{2} \beta\right)$ term. This omission neglects the interaction of waves with rotation and simplifies the remaining presentation, without any significant loss of generality. The $\mathrm{EB} \alpha$ equations corresponding to $\overline{l_{\mathrm{EB}}}$ as calculated from EP (5) are

$$
\begin{gathered}
\frac{d \mathbf{v}}{d t}+v_{j} \nabla u^{j}-\mathbf{u} \times \operatorname{curl} \mathbf{R}+g b \hat{\mathbf{z}}+\nabla \pi=0, \\
\nabla \cdot \mathbf{u}=0=\nabla \cdot \mathbf{v},
\end{gathered}
$$

where $\mathbf{v}=\mathbf{u}-\alpha^{2} \Delta \mathbf{u}, \pi=\frac{p}{\rho_{0}}-\frac{1}{2}|\mathbf{u}|^{2}-\frac{\alpha^{2}}{2}|\nabla \mathbf{u}|^{2}$, with $\mathbf{u} \cdot \hat{\mathbf{n}}=0$ and $\hat{\mathbf{n}} \times(\hat{\mathbf{n}} \cdot \nabla \mathbf{u})=0$ on the boundary, and advection dynamics for $b$ and $D$. The linear dispersion relation is

$$
\begin{aligned}
\omega^{2} & =\frac{N^{2}|\hat{\mathbf{z}} \times \mathbf{k}|^{2}+(\mathbf{k} \cdot 2 \mathbf{\Omega})^{2}}{|\mathbf{k}|^{2}\left(1+\alpha^{2}|\mathbf{k}|^{2}\right)}, \\
\text { with } N & =-\frac{g}{\rho_{0}} \frac{d \bar{\rho}(z)}{d z},
\end{aligned}
$$

where $N$ is the buoyancy frequency. Requiring the motion equation in (10) to preserve incompressibility implies a Poisson equation for the pressure $\pi$ with a Neumann boundary condition, obtained by taking the normal component of the motion equation evaluated at the boundary. Of course, the $\mathrm{EB} \alpha$ equation (10) 
reduces to the EB equation (4) when we set $\alpha=0$. Not unexpectedly, the $\mathrm{EB} \alpha$ equation is also geodesic, for a metric that involves the $H_{\alpha}^{1}$ norm [20].

Properties of $\alpha$ models. - The $\mathrm{EB} \alpha$ equation (10) conserves an energy $E_{\alpha}$ involving the $H_{\alpha}^{1}$ norm, $\|\mathbf{u}\|_{\alpha}^{2}$,

$$
\begin{aligned}
E_{\alpha} & =\int d^{3} x\left\{\rho_{0}\left(\frac{1}{2}|\mathbf{u}|^{2}+\frac{\alpha^{2}}{2}|\nabla \mathbf{u}|^{2}+g b z\right)\right\} \\
& =\frac{\rho_{0}}{2}\|\mathbf{u}\|_{\alpha}^{2}+\rho_{0} g \int d^{3} x b z .
\end{aligned}
$$

This is the sum of the Lagrangian mean kinetic and potential energies. Since $b$ is advected, the potential energy integral is bounded (for a finite domain). Consequently, conservation of $E_{\alpha}$ gives $H_{\alpha}^{1}$ control on $\mathbf{u}$, or $L^{2}$ control on $\nabla \mathbf{u}$. The curl of the $\operatorname{EB} \alpha$ motion equation yields the vorticity equation

$$
\begin{aligned}
& \frac{\partial \mathbf{q}}{\partial t}=\mathbf{q} \cdot \nabla \mathbf{u}-\mathbf{u} \cdot \nabla \mathbf{q}- g \nabla b \times \hat{z}, \\
& \text { where } \mathbf{q}=\operatorname{curl}(\mathbf{v}+\mathbf{R}) .
\end{aligned}
$$

Thus, the Helmholtz-smoothed quantity $\mathbf{u}=$ $\left(1-\alpha^{2} \Delta\right)^{-1} \mathbf{v}$ is the transport velocity for the generalized vorticity $\mathbf{q}$ and the "vortex stretching" term $\mathbf{q} \cdot \nabla \mathbf{u}$ involves $\nabla \mathbf{u}$, whose $L^{2}$ norm is controlled by the conservation of energy in Eq. (12). Boundedness of this norm will be useful in future analytical studies of the $\operatorname{EB} \alpha$ equation. For example, the filtering by the $\alpha$ term allows nonlinear Liapunov stability conditions to be formulated for equilibrium solutions of the $\mathrm{EB} \alpha$ model. In fact, Abarbanel et al. [21] introduced the notion of "conditional" Liapunov stability for the EB model, using wave number conditions that now turn out to be satisfied for the $\mathrm{EB} \alpha$ model. Equation (13) implies the potential vorticity $q_{\mathrm{EB} \alpha}=\mathbf{q} \cdot \nabla b$ is conserved along fluid trajectories, i.e., $\partial q_{\mathrm{EB} \alpha} / \partial t+\mathbf{u} \cdot \nabla q_{\mathrm{EB} \alpha}=0$. This also follows from the Kelvin-Noether circulation theorem for the $\mathrm{EB} \alpha$ model, $\frac{d}{d t} \oint_{\gamma}(\mathbf{v}+\mathbf{R}) \cdot d \mathbf{x}=-\oint_{\gamma} g z d b$, where the loop $\gamma$ moves with the transport velocity u. Hence $\mathrm{EB} \alpha$ conserves $\int d^{3} x \Phi\left(q_{\mathrm{EB} \alpha}, b\right)$ for any function $\Phi$. These are the Casimirs for the Lie-Poisson Hamiltonian formulation of the $\mathrm{EB} \alpha$ model.

When the further approximation is made that the $\mathrm{EB} \alpha$ fluid is in hydrostatic balance, i.e., that $\partial p / \partial z+\rho_{0} g b=$ 0 , then we find a new primitive-equation- $\alpha$ model (PE $\alpha$ ). We expect this PE $\alpha$ model to be useful for numerically simulating large-scale weather patterns and global winddriven ocean circulation over long times, because its linear and nonlinear dispersion filters out rapid gravity wave fluctuations while taking their mean effects into account at coarse scales $L$, where $\alpha / L \ll 1$. We address the threedimensional mechanism of this nonlinear dispersion next.

n-dimensional CH. - Ignoring buoyancy and rotation terms in $\overline{l_{\mathrm{EB}}}$ in Eq. (9) removes the linear dispersion and gives the Lagrangian for the following $n$-dimensional incompressible generalization of the $\mathrm{CH}$ equation (1),

$$
\begin{gathered}
\frac{\partial \mathbf{v}}{\partial t}+\mathbf{u} \cdot \nabla \mathbf{v}+v_{j} \nabla u^{j}+\nabla \pi=0, \\
\nabla \cdot \mathbf{u}=0=\nabla \cdot \mathbf{v},
\end{gathered}
$$

where $\mathbf{v}=\mathbf{u}-\alpha^{2} \Delta \mathbf{u}$,

$$
\pi=\frac{p}{\rho_{0}}-\frac{1}{2}|\mathbf{u}|^{2}-\frac{\alpha^{2}}{2}|\nabla \mathbf{u}|^{2} .
$$

Equations of the type (14) but with additional dissipative terms were considered previously in the theory of second grade fluids [22] and were treated recently in the mathematical literature [23,24]. Second grade fluid models are derived from continuum mechanical principles of objectivity and material frame indifference, after which thermodynamic principles such as the Clausius-Duhem relation and stability of stationary equilibrium states are imposed that restrict the allowed values of the parameters in these models. In contrast, the $\mathrm{CH}$ equation (14) is derived here by applying asymptotic expansions, Lagrangian means, and an assumption of isotropy of fluctuations in Hamilton's principle for an ideal incompressible fluid. This derivation provides the interpretation of $\alpha$ as the typical amplitude of the rapid fluctuations over whose phase the Lagrangian mean is taken in Hamilton's principle.

The $n$-dimensional $\mathrm{CH}$ equation (14) is geodesic on the volume-preserving diffeomorphisms with the $H_{\alpha}^{1}$ norm. Its conservation laws include energy $\frac{1}{2} \int d^{3} x \mathbf{u} \cdot \mathbf{v}$ and helicity $\frac{1}{2} \int d^{3} x \mathbf{v} \cdot \operatorname{curl} \mathbf{v}$. Its steady vortical flows include the analogs of the Beltrami flows curl $\mathbf{v}=\lambda \mathbf{u}$. In the periodic case, we define $\mathbf{v}_{\mathbf{k}}$ as the $\mathbf{k}$ th Fourier mode of the specific momentum $\mathbf{v} \equiv\left(1-\alpha^{2} \Delta\right) \mathbf{u}$, so that $\mathbf{v}_{\mathbf{k}} \equiv\left(1+\alpha^{2}|\mathbf{k}|^{2}\right) \mathbf{u}_{\mathbf{k}}$. Then Eq. (14) becomes

$$
\Pi_{\perp}\left(\frac{d}{d t} \mathbf{v}_{\mathbf{k}}-i \sum_{\mathbf{p}+\mathbf{n}=\mathbf{k}} \frac{\mathbf{v}_{\mathbf{p}}}{1+\alpha^{2}|\mathbf{p}|^{2}} \times\left(\mathbf{n} \times \mathbf{v}_{\mathbf{n}}\right)\right)=0,
$$

where $\Pi_{\perp}$ is the Leray projection onto Fourier modes transverse to $\mathbf{k}$ (this ensures incompressibility). Hence, the nonlinear coupling among the modes is suppressed by the denominator when $1+\alpha^{2}|\mathbf{p}|^{2} \gg|\mathbf{n}|$.

An essential idea of the $n$-dimensional $\mathrm{CH}$ equation (14) is that its specific momentum $\mathbf{v}$ is transported by a velocity $\mathbf{u}$ which is smoothed, or filtered, by inverting the elliptic Helmholtz operator $\left(1-\alpha^{2} \Delta\right)$. The effect on length scales smaller than $\alpha$ is that steep gradients of the specific momentum $\mathbf{v}$ tend not to steepen much further, and thin vortex tubes tend not to get much thinner as they are transported. And, as numerical simulations verify [25], the effect on length scales larger than $\alpha$ is negligible. This is also borne out for vortex interaction in two dimensions [26]. Hence, the $n$-dimensional $\mathrm{CH}$ equation and the other $\alpha$ models preserve the assumptions under which they are derived. One may also formulate the $n$-dimensional $\mathrm{CH}$ equation on a general Riemannian manifold. The formulation of $\mathrm{CH}$ as a geodesic spray equation on a Riemannian manifold is useful in studying certain analytical properties 
of its solutions [27]. Finally, the formulation of compressible $\alpha$ models is straightforward; see [15].

We thank R. Camassa, S. Y. Chen, P. Constantin, C. Doering, C. Foias, R. Kinney, J.C. McWilliams, V. L'vov, A. Mahalov, E. Titi, and V. Zeitlin for their time, encouragement, and valuable input. Work by D. H. was conducted under the auspices of the U.S. Department of Energy. Work of J. M. was supported by the California Institute of Technology and NSF Grant No. DMS 9633161. Work by T.R. was partially supported by NSF Grant No. DMS-9503273 and DOE Contract No. DEFG03-95ER25245-A000.

*Electronic address: dholm@lanl.gov

†Electronic address: marsden@cds.caltech.edu

†Electronic address: ratiu@math.ucsc.edu

[1] I. Gjaja and D. D. Holm, Physica (Amsterdam) 98D, $343-$ 378 (1996).

[2] R. Camassa and D. D. Holm, Phys. Rev. Lett. 71, 16611664 (1993).

[3] R. Camassa, D. D. Holm, and J. M. Hyman, Advances in Applied Mechanics (Academic Press, Boston, 1994), Vol. 31, pp. 1-33.

[4] M. Alber, R. Camassa, D. D. Holm, and J. E. Marsden, Lett. Math. Phys. 32, 137-151 (1994).

[5] M. Alber, R. Camassa, D. D. Holm, and J. E. Marsden, Proc. R. Soc. 450, 677-692 (1995).

[6] F. Calogero, Phys. Lett. A 201, 306-310 (1995).

[7] F. Calogero and J. P. Francoise, J. Math. Phys. (N.Y.) 37, 2863-2871 (1996).

[8] O. Ragnisco and M. Bruschi, Physica (Amsterdam) 228A, 150-159 (1996).

[9] Y. B. Suris, Phys. Lett. A 217, 321-329 (1996).

[10] B. Fuchssteiner, Physica (Amsterdam) 95D, 229-243 (1996).

[11] J. Schiff, "The Camassa-Holm Equation: A Loop Group Approach" (to be published).

[12] G. Misiolek, "A Shallow Water Equation as a Geodesic
Flow on the Bott-Virasoro Group" (to be published).

[13] The $\kappa \neq 0$ case is slightly more technical; see [12].

[14] J.E. Marsden and T.S. Ratiu, Introduction to Mechanics and Symmetry, Texts in Applied Mathematics Vol. 17 (Springer, New York, 1994).

[15] D. D. Holm, J.E. Marsden, and T.S. Ratiu, "EulerPoincaré Equations and Semidirect Products with Applications to Continuum Theories," Adv. Math. (to be published).

[16] J. Pedlosky, Geophysical Fluid Dynamics (Springer, New York, 1987), 2nd ed.

[17] V.I. Arnold, Ann. Inst. Fourier, Grenoble 16, 319-361 (1966).

[18] The metric for which the EB equations are geodesic is $\int d^{3} x\left[|\mathbf{u}|^{2}+\left(\omega_{1}+\mathbf{u} \cdot \mathbf{R}\right)^{2}+\left(\omega_{2}-g z\right)^{2}\right]$ where $\omega_{1,2}=d \theta_{1,2} / d t$, for gauge fields $\theta_{1}, \theta_{2}$, and $\frac{d}{d t}\left(\omega_{1}+\right.$ $\mathbf{u} \cdot \mathbf{R})=0=\frac{d}{d t}\left(\omega_{2}-g z\right)$; see [15].

[19] D. G. Andrews and M.E. McIntyre, J. Fluid Mech. 89, 609-646 (1978).

[20] The metric for which the $\operatorname{EB} \alpha$ equations are geodesic is $\|u\|_{\alpha}^{2}+\int d^{3} x\left[\left(\omega_{1}+\mathbf{u} \cdot \mathbf{R}\right)^{2}+\left(\omega_{2}-g z\right)^{2}\right]$; see [18].

[21] H. D. I. Abarbanel, D.D. Holm, J.E. Marsden, and T. Ratiu, Philos. Trans. R. Soc. London A 318, 349-409 (1986).

[22] J. E. Dunn and R. L. Fosdick, Arch. Ration. Mech. Anal. 56, 191-252 (1974).

[23] D. Cioranescu and V. Girault, C. R. Acad. Sci. Paris Série 1 322, 1163-1168 (1996).

[24] D. Cioranescu and V. Girault, Int. J. Non-Linear Mech. 32, 317-335 (1997).

[25] S. Y. Chen, C. Foias, D. D. Holm, E. Olson, E. S. Titi, and S. Wynne, "The Steady Viscous Camassa-Holm Equations as a Reynolds Algebraic Stress Closure Model for Turbulent Channel Flows and Their Boundary Layer Analysis" (to be published).

[26] D. D. Holm, R. Kinney, and J.C. McWilliams, "Vortex Interaction in Planar $\alpha$ Models" (to be published).

[27] D. D. Holm, S. Kouranbaeva, J.E. Marsden, T. Ratiu, and S. Shkoller, "Euler-Poincaré Equations for Continuum Theories on Riemannian Manifolds" (to be published). 